\title{
Development of UAV Teleoperation Virtual Environment Based-on GSM Networks and Real Weather Effects
}

\author{
Amr AbdElHamid* \\ College of Electronic and Information Engineering, Nanjing University of Aeronautics and Astronautics (NUAA), 29 Yudao \\ Street, Nanjing 210016, China.
}

Peng Zong

Astronautics College, NUAA, Nanjing 210016, China.

\begin{abstract}
Future Ground Control Stations (GCSs) for Unmanned Aerial Vehicles (UAVs) teleoperation targets better situational awareness by providing extra motion cues to stimulate the vestibular system. This paper proposes a new virtual environment for long range Unmanned Aerial Vehicle (UAV) control via Non-Line-of-Sight (NLoS) communications, which is based on motion platforms. It generates motion cues for the teleoperator for extra sensory stimulation to enhance the guidance performance. The proposed environment employs the distributed component simulation over GSM network as a simulation platform. GSM communications are utilized as a multi-hop communication network, which is similar to global satellite communications. It considers a UAV mathematical model and wind turbulence effects to simulate a realistic UAV dynamics. Moreover, the proposed virtual environment simulates a Multiple Axis Rotating Device (MARD) as Human Machine Interface (HMI) device to provide a complete delay analysis. The demonstrated measurements cover Graphical User Interface (GUI) capabilities, NLoS GSM communications delay, MARD performance, and different software workload. The proposed virtual environment succeeded to provide visual and vestibular feedbacks for teleoperators via GSM networks. The overall system performance is acceptable relative to other Line-of-Sight (LoS) systems, which promises a good potential for future long range, medium altitude UAV teleoperation researches.
\end{abstract}

Key words: UAV teleoperation, Virtual environment, Wind turbulence, GSM communications.

\section{Introduction}

During the last decades, Research and Development (R\&D) facilities provide a remarkable interest in Unmanned Aerial System (UAS) in all fields such as flight simulators [13], different UAV development scales [4, 5], Ground Control Stations (GCSs) [6]... etc. In spite of the great success of UAVs autonomous flight control missions (in civilian and military sections), they are equipped with high technology components which could be potentially subject to cyberattack with serious repercussions (like spoofing threats [7, 8]). Wherefore, the remotely piloted mission is not obsolete, especially when threats exist against UAVs during missions. Pilots in cockpits are considered as a key factor of any mission success, due to their doubtless intelligent responses towards encountering any expected or unexpected situation. Due to unsafe nature of missions, UAV teleoperators can perform Dull, Dirty, and Dangerous (3D) missions safely using GCS. Situational awareness of teleoperators in UAV controlled missions, particularly for Non-Line-of-Sight (NLoS) missions, is of foremost importance due to increased latency induced by multi-hop data links.

For enhancing human operator's performance, extra stimulation has emerged in many applications such as UAV
This is an Open Access article distributed under the terms of the Creative Commons Attribution Non-Commercial License (http://creativecommons.org/licenses/by$\mathrm{nc} / 3.0 /$ which permits unrestricted non-commercial use, distribution, and reproduction in any medium, provided the original work is properly cited.

\footnotetext{
(c) * Doctoral Candidate, Corresponding Author: Amr@nuaa.edu.cn

** PH.D. from United Kingdom, Professor of NUAA
} 
teleoperation, under-water tasks [9, 10], obstacle avoidance through haptic interfaces [11-13], and ground robots tasks $[14,15]$. Moreover, Max Planck Institute (MPI) introduced technical issues to use KAKU Robocoaster for use as a realtime motion simulator [16]. A motion platform is utilized to augment pilot performance and assesses UAV pilot skills using a simulator system [17]. A CyberMotion simulator is utilized to integrate vestibular feedback with the usual visual feedback obtained from a UAV onboard camera [18]. Mixed reality is introduced by integrating real UAV camera frames into a virtual world in near earth environment using a motion simulator [19]. In [20], a teleoperation using Google glass is introduced to control a small scale UAV through the operator interaction events (gesture and head motion). Finally, different MARD platforms are utilized by different applications for various purposes such as: video gaming and entertainments, medical treatments, and simulated training. In [21], a computer-controlled experiment is conducted for 25 participants using a multi-axis chair; such chair can perform rotation combinations of whole body pitch and yaw simultaneously. The experiment results show that participants' stability is improved and sway is reduced. In this paper, MARD platform in [21] is employed for a new area of application (virtual environments for UAV teleoperations), and thereafter for real teleoperation missions.

Some of the shortcomings in aforementioned research work are: (1) utilizing only line of sight data communication links for UAV feedbacks. (2) Missing real wind parameters at every zone of the UAV mission inside the teleoperation workstation (short range missions only are considered). (3) Limiting the conducted mission range to the range of Remote Control (RC) transmitter.

Thus, the main objectives of this paper are to:

1. Propose a new virtual environment for UAV teleoperation, to enhance human operator performance through visual and vestibular feedbacks for better situational awareness.

2. Propose a distributed component simulation platform based on real NLoS communications.

3. Include realistic wind turbulence effects, which are based- on real weather forecast data at every zone along the UAV mission.

4. Demonstrate a delay analysis for the proposed platform based-on real GSM communication channels.

Towards fulfilling the aforementioned objectives, the proposed virtual environment is developed to simulate a teleoperation GCS, which provides a visual and vestibular cues for human operator. Such cues enhance the teleoperator performance and increase the situational awareness during different UAV missions. As a result, more mission success ratio and less hazardous are obtained. In other words, the UAV teleoperator body rolls when the UAV rolls, and pitches when the UAV performs a pitch maneuver, for more realistic responses and reflexes. This can be performed by using a hydraulic or electric chair, which has rotational degrees of freedom. Multiple Axis Rotating Devices (MARDs) are used for simulated training, gaming/entertainments, and medical treatments [21]. Furthermore, MARD dynamics is controllable to provide the maximum real situation with safe rotational rates for the pilot body $[21,22]$. Through this paper, MARD is proposed to compete in real UAV guidance operations. Moreover, synchronizing MARD motion with real UAV attitude could be enhanced via time-stamped data, and channel propagation delay estimation.

For both civilian and military scenarios, the proposed virtual environment provides the following advantages: (1) It increases teleoperators situational awareness to minimize UAVs accidents. (2) It allows new types of long range UAV missions. (3) It facilitates realistic testing of medium altitude Unmanned Combat Aerial Vehicles (UCAV) devices. (4) It provides more flexibility for evaluating different UAV's performance by changing the backbone mathematical model. (5) It facilitates realistic dynamics during UAV controlled missions by including real captured weather data into the simulation loop. (6) It enhances teleoperators experience to combat realistic weather conditions, which is sometimes beyond autopilot capability. (7) It opens new prospect for different MARD manufacturers to compete in real controlled missions. (8) It can be upgraded to a functional UAV teleoperation GCS with minor modifications.

The results show that the proposed virtual environment succeeded to execute a simulated mission via real GSM communication channels, while the developed simulated MARD model fulfills all UAV maneuvers in a realistic wind turbulence conditions. Furthermore, the proposed system achieves an acceptable performance using NLoS communication relative to other systems based on Line-ofSight (LoS) data links.

Many difficulties have conquered during the development of proposed virtual environment such as: (1) designing a virtual environment with a realistic topology, which facilitates future upgrading to a real UAV teleoperation GCS. (2) Involving real multi-hop communication channels into the simulation loop to achieve realistic measurements. (3) Injecting real weather data into UAV simulated missions to achieve realistic unstable dynamics. (4) Developing a precise data logging module for advanced evaluation and playback capabilities. 
Unlike [1-3, 16-18, 23], the proposed environment (1) Provides advanced realistic UAV simulated missions with visual and vestibular motion effects. (2) Utilizes realistic topologies to facilitate the upgrading to a real long range teleoperation GCS. (3) It exploits a real NLoS communication channel for uplink and downlink data. (4) It facilitates better situational awareness about UAV attitude along the mission. (5) It exploits a global weather forecast data to enhance mission planning and UAV guidance operations. (6) In addition to NLoS capabilities, it achieves less delay than other systems.

Therestofthispaperis organized asfollow:Section 2 presents the modeling process of converting a real teleoperation model to the proposed virtual environment. Section 3 details the proposed virtual environment development components, while Sections 4 and 5 comprehensively present the results and the qualitative aspects of the proposed virtual environment with other systems. Finally, the conclusions are presented in Section 6 .

\section{Proposed Virtual Environment Framework Modeling}

This section presents the modeling steps to convert a real teleoperation scenario into a virtual environment.

Fig. 1 represents a realistic teleoperation framework for different types of UAVs using various MARDs through diverse communication channels. The rotation rates and angles already transmitted to the GCS for visualization through gauges. However, these data are utilized to control MARD for extra sensory stimulation for UAV teleoperators. Furthermore, the received attitude data include the effects of wind turbulence which affect UAVs in different mission. All these dynamics are realized by teleoperator using any MARD as illustrated in Fig. 1. Hence, teleoperator picks up the rotation rates and angles and uses these cues to improve the performance. It is obviously noticed from Fig. 1 that providing a heavy payload MARD requires quite large budget. Therefore, before affording a quiet large budget to provide

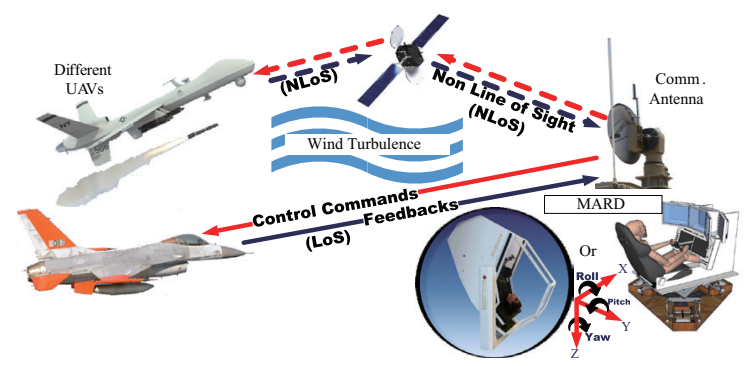

Fig. 1. Different Scenarios for Realistic UAV Teleoperation Framework a MARD for UAV teleoperation research, it is important to assure its characteristics in simulated UAV missions. Thus, an attempt is conducted to build a virtual environment to consider Fig. 1 components.

As shown in Fig. 2, the simulated platform consists of two workstations, workstation 1 represents the airborne vehicle and its surrounding environment, while workstation 2 acts as a teleoperation workstation to control such UAV. Workstation 1 hosts MATLAB and equipped with the Flight Dynamics and Control (FDC) toolbox [24] to provide UAV dynamics to the system. In addition, Dryden wind turbulence model is integrated with the UAV mathematical to generate stochastic linear and angular wind components to imitate the wind turbulence in Fig. 1.

Moreover, workstation 1 contains a background application which is so-called MATLAB interface module. This module is responsible of capturing the GCS commands from the GSM modem, executes these commands in the MATLAB simulation models and returns the feedback results. This module utilizes the MATLAB Component Object Model (COM) automation server $[25,26]$. On the other hand, workstation 2 hosts the GCS graphical user interface, which is responsible to interface the RC transmitter, global weather forecast providers, the real GSM modem and the simulated 3D MARD model according to the specifications in [21]. Through this virtual environment, the teleoperator can control the Beaver aircraft and recognize its dynamics through onscreen gauges and through the MARD simulated model. This scenario is conducted using NLoS communications via the exploitation of GSM modems. The proposed virtual environment topology facilitates the future upgrading to a real UAV GCS, as by replacing workstation 1 with a real UAV prototype and minor software modification, the whole

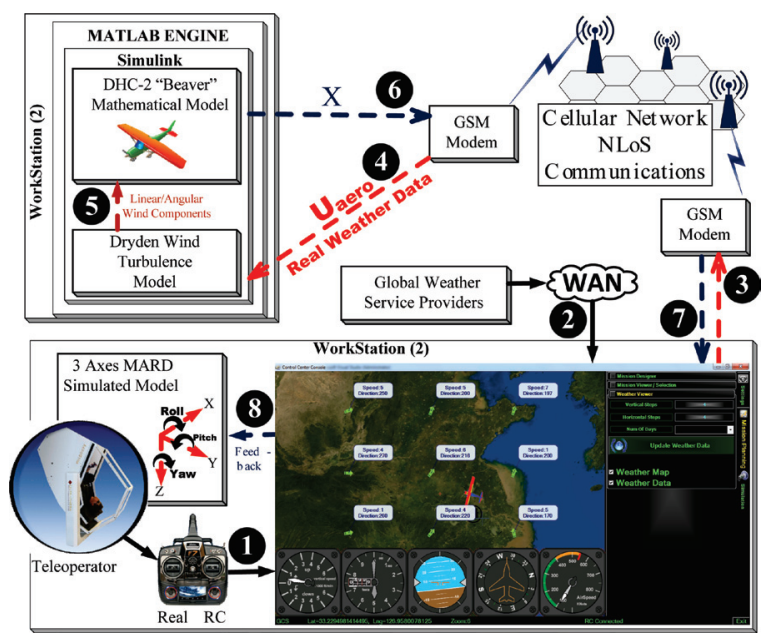

Fig. 2. Proposed UAV Virtual Environment using Real NLoS GSM Link 
remaining infrastructure, interfaces, and modules can work as a real UAV teleoperation workstation with visual and vestibular feedbacks.

\section{Proposed Virtual Environment Compo- nents and Methodologies}

This section covers a comprehensive component development of the proposed virtual environment ranging from different mathematical models to the GSM bidirectional communication.

\subsection{Backbone Aircraft Mathematical Model}

The proposed virtual environment utilizes FDC toolbox to achieve the UAV dynamics [24]. The basic Newtonian mechanics are used to derive the aircraft equation of motion, starting with the rigid body force and the moment equations which are stated as follow [24]:

$$
\begin{aligned}
& \boldsymbol{F}=m\left(\frac{\partial \boldsymbol{V}_{A}}{\partial t}+\boldsymbol{\Omega}_{A} \times \boldsymbol{V}_{A}\right) \\
& \boldsymbol{M}=\frac{\partial\left(\boldsymbol{I} \cdot \boldsymbol{\Omega}_{A}\right)}{\partial t}+\boldsymbol{\Omega}_{A} \times\left(\boldsymbol{I} . \boldsymbol{\Omega}_{A}\right)
\end{aligned}
$$

where $m$ is the aircraft mass, $\boldsymbol{V}_{A}=\left[\begin{array}{lll}u & v & w\end{array}\right]^{T}$ is the aircraft velocity vector at the center of gravity (c.g.) where $u, v, w$ are the velocity component along the $X_{\text {Body }}$ axis, $Y_{\text {Body }}$ axis, and $Z_{\text {Body }}$ axis, respectively. $\boldsymbol{\Omega}_{A}=[p q r]^{T}$ is the aircraft angular velocity vector about the c.g. where $p$ is the angular rate of roll, $q$ is the angular rate of pitch, and $r$ is the angular rate of yaw, $\boldsymbol{F}=\left[\begin{array}{lll}F_{x} & F_{y} & F_{z}\end{array}\right]^{T}$ is the total external force vector where $F_{x}$, $F_{y}, F_{z}$ are the total external force along $X_{B o d y}$-axis, $Y_{B o d y}$-axis, and $Z_{B o d y}$-axis, respectively. $M=[L M N]^{T}$ is the total external moment vector where $L, M, N$ are total rolling, pitching, and yawing moments, respectively, and I is the inertia tensor of the rigid body. Derivation is continued till reaching the external aerodynamic control inputs vector $\mathbf{u}_{\text {aero }}$ and output state vector $\boldsymbol{X}$ as follow [24]:

$$
\begin{aligned}
& \mathbf{u}_{\text {aero }}=\left[\begin{array}{llll}
\delta_{e} & \delta_{a} & \delta_{r} & \delta_{f}
\end{array}\right]^{T} \\
& \mathbf{X}=\left[\mathrm{V}, \alpha, \beta, p, q, r, \psi, \theta, \varphi, x_{e}, y_{e}, H\right]^{T}
\end{aligned}
$$

where, $\mathbf{X}$ is the aircraft mathematical model output state vector, which contains twelve elements: true airspeed $(V)$, angle of attack $(\alpha)$, and sideslip angle $(\beta)$, three angular velocities $(p, q, r)$, three Euler angles which define the attitude of the aircraft relative to the Earth $(\psi, \theta, \varphi)$, two coordinates and the altitude which define the position of the aircraft $\left(x_{e}, y_{e}, H\right)$ relative to the Earth.

\section{2 Atmospheric Wind Turbulence Model}

Numerical methods are used to generate the linear and angular velocities of atmospheric turbulence to conduct numerical simulations. Dryden model is considered as one of the most attractive models for atmospheric turbulence, due to the ability of factorizing the Dryden atmospheric turbulence rational formula (which is necessary for the numerical simulations). Thus, this model is utilized in this paper. The Dryden model is a function form that empirically fits the measured power spectral data. It handles the linear and angular velocity components of the continuous gusts as random processes. This model assumes that turbulent gusts are isotropic (the statistical properties do not depend upon the spatial orientation of air mass itself) [27]. The Dryden model is described by Power Spectral Densities (PSDs) for wind gusts three linear velocity components $\left(u_{w}, v_{w}\right.$ and $\left.w_{w}\right)$ which can be expressed as follow [28]:

$$
\begin{gathered}
\Phi_{u_{w}}(\Omega)=\frac{2 \sigma_{u_{w}}^{2} L_{u_{w}}}{\pi} \cdot \frac{1}{1+\left(L_{u_{w}} \Omega\right)^{2}} \\
\Phi_{v_{w}}(\Omega)=\frac{\sigma_{v_{w}}^{2} L_{v_{w}}}{\pi} \cdot \frac{1+3\left(L_{v_{w}} \Omega\right)^{2}}{\left[1+\left(L_{v_{w}} \Omega\right)^{2}\right]^{2}} \\
\Phi_{w_{w}}(\Omega)=\frac{\sigma_{w_{w}}^{2} L_{w_{w}}}{\pi} \cdot \frac{1+3\left(L_{w_{w}} \Omega\right)^{2}}{\left[1+\left(L_{w_{w}} \Omega\right)^{2}\right]^{2}}
\end{gathered}
$$

where $\sigma_{j}$ and $L_{j}$ are the turbulence intensity and scale length, respectively, for the $j^{\text {th }}$ velocity component, and $\Omega$ is a spatial frequency $(\mathrm{rad} . / \mathrm{m})$ [29]. The circular frequency $\omega$ can be defined as:

$$
\omega=\Omega \cdot V
$$

where $V$ is speed of UAV flying through a frozen turbulence field. Using the wind gust angular velocity components ( $p_{w}$, $\left.r_{w}, q_{w}\right)$, the power spectral densities for these components can be expressed as [30]:

$$
\Phi_{p_{w}}(\omega)=\frac{\sigma_{w_{w}}^{2}}{V L_{w_{w}}} \cdot \frac{0.8\left(\frac{\pi L_{w_{w}}}{4 b}\right)^{1 / 3}}{1+\left(\frac{4 b \omega}{\pi V}\right)^{2}}
$$

$\Phi_{r_{w}}(\omega)=\frac{\mp\left(\frac{\omega}{V}\right)^{2}}{1+\left(\frac{3 b \omega}{\pi V}\right)^{2}} \cdot \Phi_{v_{w}}(\omega)$

$\Phi_{q_{w}}(\omega)=\frac{ \pm\left(\frac{\omega}{V}\right)^{2}}{1+\left(\frac{4 b \omega}{\pi V}\right)^{2}} \cdot \Phi_{w_{w}}(\omega)$ 
where $\Phi_{i}(\Omega)=V \Phi_{i}\left(\frac{\omega}{V}\right)$, and $b$ represents the UAV wingspan.

However, to achieve realistic wind turbulence from the above model, real weather parameters must be supplied as inputs. The proposed virtual environment exploits "WorldWeatherOnline" as a worldwide instantaneous weather provider (as a sample to validate the concept). The weather provider online Application Programming Interface (API) explains the structure and formats of weather data exchange service. Therefore, the proposed environment captures real weather data over internet, and transmits these data over GSM modems to workstation 2 to supply Dryden wind turbulence module with real wind parameters, which will generate realistic linear and angular wind components for the UAV mathematical model. As a result, the Dryden wind turbulence model can generate a realistic wind turbulence according to real weather forecast data. As shown in Fig. 3, a real weather data (north wind with $6 \mathrm{~m} / \mathrm{s}$ ) is converted to a complete set of linear and angular wind velocities in consideration of the UAV altitude. At $6000 \mathrm{ft}$ altitude, the wind linear velocity components randomly vary between -3.5 to $+2.8 \mathrm{~m} / \mathrm{s}$, while the angular velocities components vary stochastically between -0.07 to $+0.07 \mathrm{rad} / \mathrm{s}$

Moreover, the proposed real weather forecast addon service is exploited also during mission planning and guidance operation phases. Fig. 4 (a) demonstrates a detailed flowchart of the proposed weather interface module (which is implemented as a standalone thread). This module is responsible of web communication with weather providers through the implementation of provider's APIs. Furthermore, this module parses the provider responses into useful information and stores them into in-memory data base. However, Fig. 4 (b) represents useful weather data and its effect on the mission planning (for long range UAVs). It can be deduced that the overlaid weather layer supports mission planner operator to setup a safe trajectory
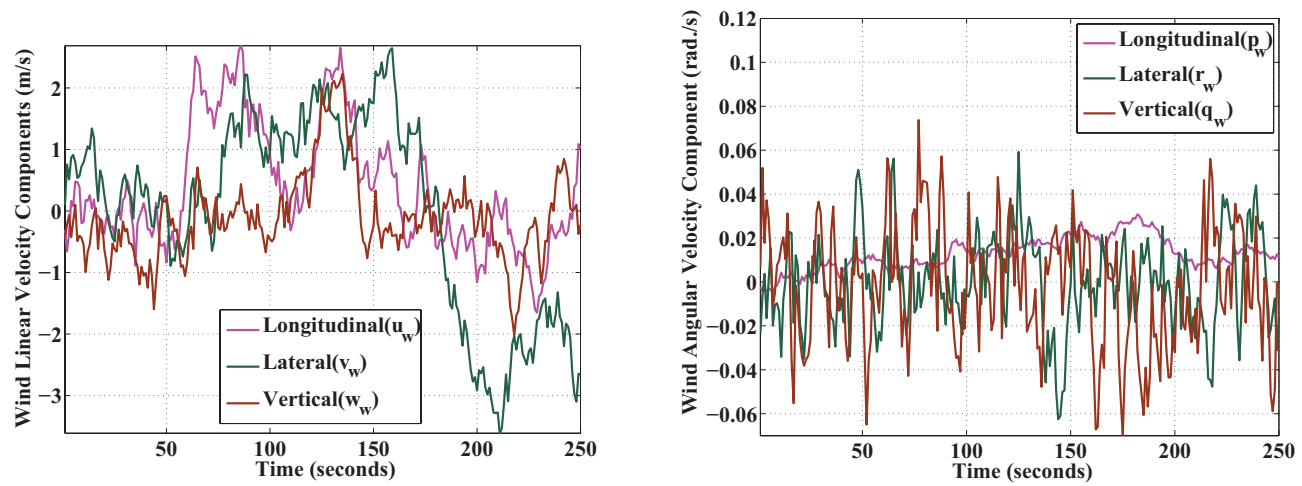

Fig. 3. Random Generation of Wind Velocities Based on Real Weather Reports (a) Linear Components, (b) Angular Components
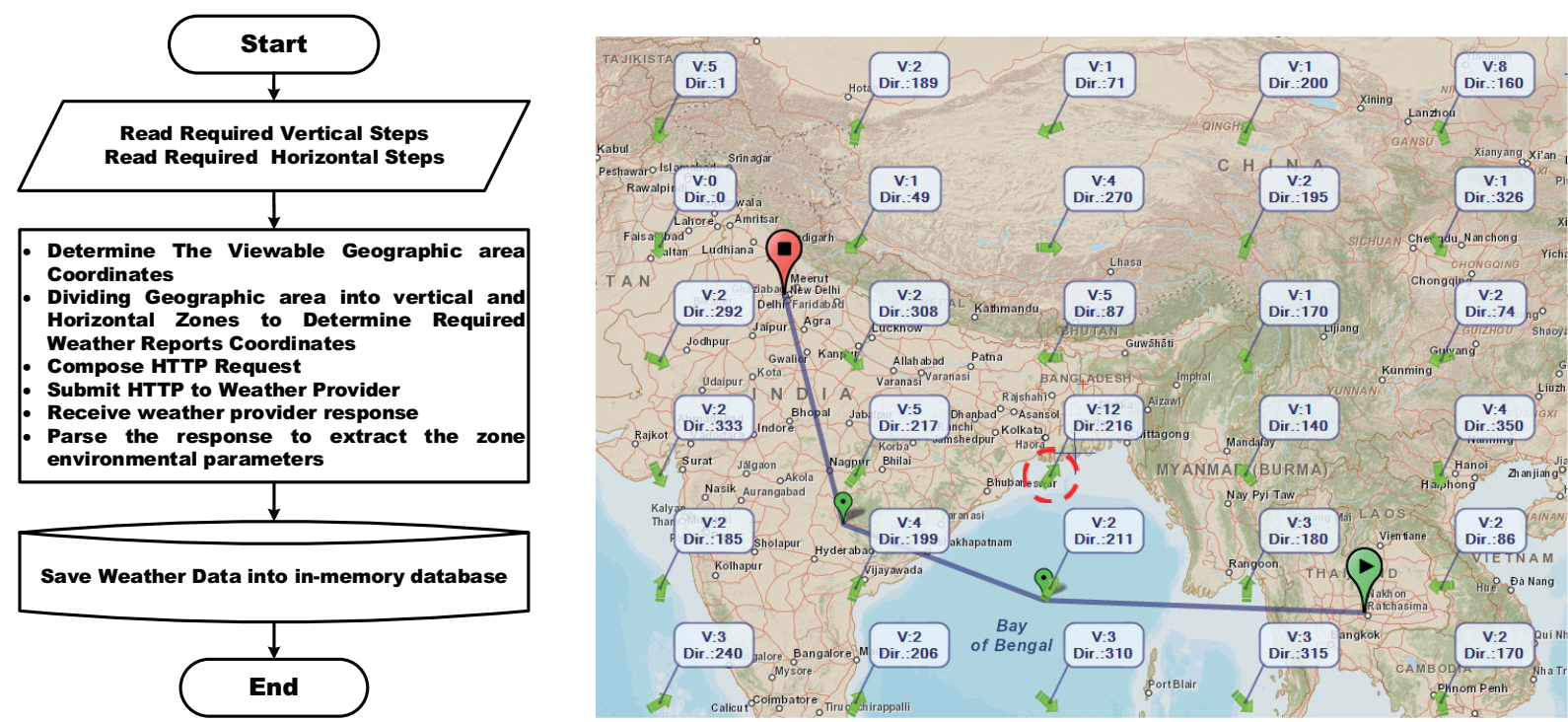

Fig. 4. Integration of Global Weather Forecasting Reports (a) Weather Interface Module Flowchart, (b) Mission Planning using Synthetic Weather Layer 
for the mission. Because of existence of such weather layer, the mission planner avoids a side wind of speed $12 \mathrm{~m} / \mathrm{sec}$ coming from direction 216 (which is illustrated in red dashed circle).

\subsection{Multiple Axis Rotating Device Model}

Usually, MARDs are a fully automated and computer controlled equipment, they can be used for entertainment and gaming, simulated training, and medical treatments (like motion sickness desensitization and postural stability). In Ref. [21], a computer-controlled multi-axis chair is used to improve the stability of patients through whole patient body rotation at $90 \mathrm{deg}$./sec. (15 RPM). Typically, this kind of MARD is suitable to be considered for the proposed virtual environment, due to its heavy payload and its high angular rotation rates. It is worthily noted that pan, tilt, and roll rotation rates are extracted from Ref.[21], which is assumed to have its own built in washout filters. The MARD motors angles will be noted as pan, tilt, roll angles which represents the aircraft yaw, pitch, and roll angles $(\psi, \theta$, and $\varphi)$.

In general, the transfer function of the second order 3D
MARD platform can be written as follow:

$$
\begin{aligned}
& G_{i}(s)=\frac{Y_{i}(s)}{U_{i}(s)} \\
& =\frac{k_{i} \omega_{n_{i}}}{s^{2}+2 \xi_{i} \omega_{n_{i}} s+\omega_{n_{i}}{ }^{2}} ; i=\{\psi, \theta, \varphi\}
\end{aligned}
$$

where: $i$ represent yaw $(\psi)$, pitch $(\theta)$ or roll $(\varphi)$ rotational axes for different motors of the MARD platform, $Y_{i}(s), U_{i}(s)$ are Laplace transforms of pan, tilt, or roll executed angles $y_{i}(t)$ and the commanded yaw, pitch, or roll angles $u_{i}(t)$, respectively. $k_{i}, \xi_{i}$, and $\omega\left(n_{i}\right)$ are gain, damping ratio, and natural frequency of different rotating axis $i$, respectively.

It is clear that eq. (12) is valid for any MARD platform regardless its payload. Therefore, the above equation is represented in Simulink model as a nonlinear second order actuators. This representation facilitates the study of demanded MARD platform in pan, tilt, and roll directions (which is considered one of the conquered difficulties in this paper).

\subsection{Non Line of Sight Bidirectional GSM Communi- cations}

The GSM data communications could be established through many setup profiles. The proposed platform

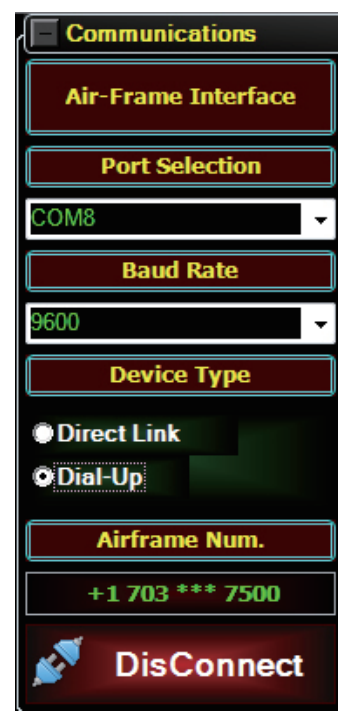

(a)

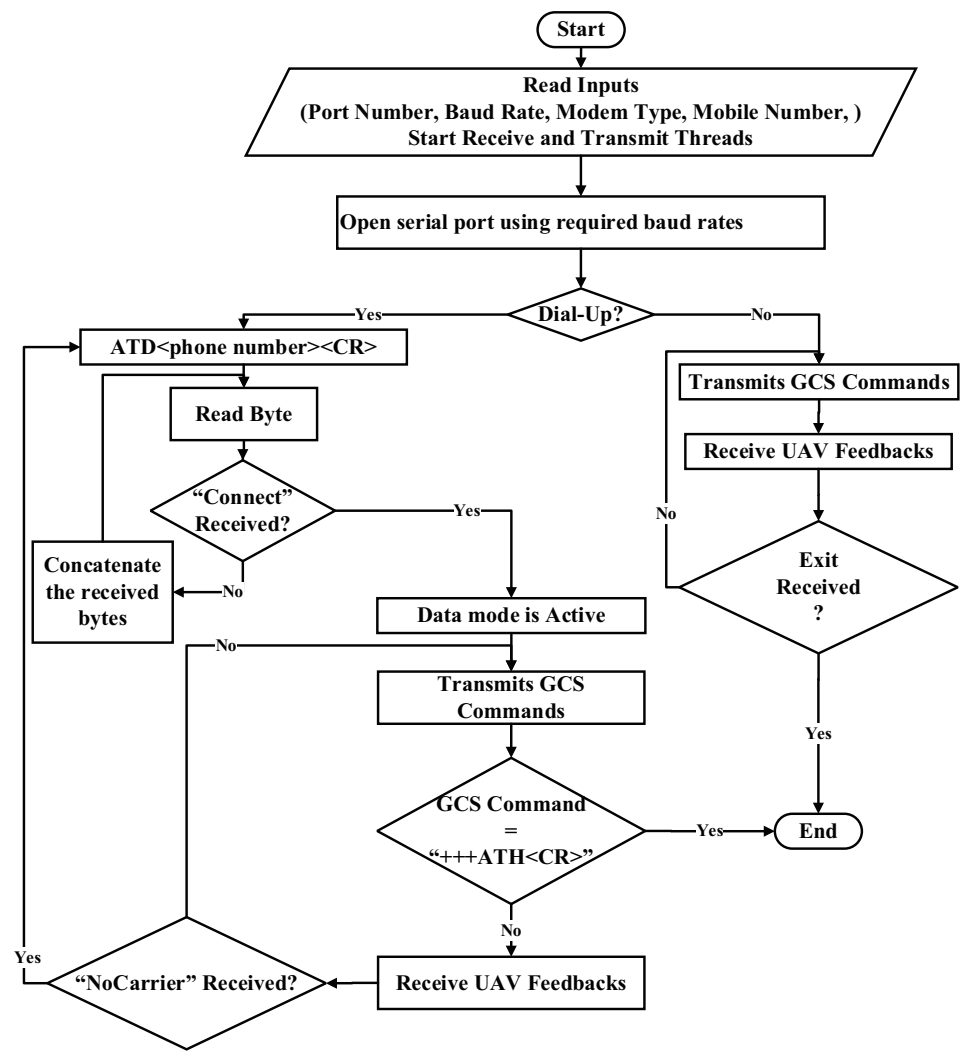

(b)

Fig. 5. Ground GSM Communication Interface (a) GSM Controls (b) Detailed Flowchart 
utilizes Point to Point Protocol (PPP) for controlling the UAV. PPP mode consists of two communication parties, one of them is the master (who initiates a communication request), while the other one is slave (who answers the call). Typically, through the standard AT commands [31], the GCS can play master role and can initiate the communication request. The GSM call routing from ground PC-based pilot is initiated with the following AT dial command: "ATD $<$ phone number $><\mathbf{C R}>$ ". This call is routed to the airborne GSM modem which is identified by Subscriber Identity Module (SIM). The airborne GSM modem (which is connected to Workstation 2) performs a compatibility check then returns a call-confirmed message master modem. Once the call confirmationmessageis received, themobile communication enters the active state to exchange bidirectional data. On the other hand, for terminating this connection, it is required to exit the data mode first using the command "+++", followed by the AT hang command: "ATH”. As shown in Fig. 5 (a), the Graphical User Interface (GUI) receives the perquisite parameters for establishing the communication with the UAV mathematical model. This communication supports both dial up modems and leased line ones, while Fig. 5 (b) illustrates the flowchart for such module. It can be inferred that handling the dial up connections requires more programming effort and sensitive data handling (rather than the leased line or wireless modem), due to the switching between the data and commands modes and to detect any disconnect event.

Moreover, at the workstation 2, when the GCS call event is detected, the developed avionics accept the call by issuing the following answer command "ATA $<\mathbf{C R}>$ ", once the communication link is active, all teleoperator commands and real weather forecast data are transported to workstation 2, and UAV feedback are transmitted back to the teleoperation workstation.

\subsection{Proposed Virtual Environment Framework Inte- gration}

In Fig. 2, the proposed simulated platform captures real weather data using global weather providers API and displays them as synthetic data layer over GUI. The GUI sets the parameters of Dryden wind model (on workstation 1) with the captured real weather conditions to generate realistic wind turbulence components. Within the Simulink environment, generated wind turbulence parameters are propagated to Beaver model with the RC guidance commands to generate a realistic "Beaver" dynamics. The "Beaver" output state vector $\mathbf{X}$ is transmitted back through GSM channels to the GCS (to consider a practical channel propagation delay), and finally, the feedbacks are propagated to the 3D MARD model to simulate the real MARD dynamics. Providing such feedback to the simulated MARD model lets the latter act as if it is an UAV cockpit. Workstation 1 activities is executed using the active hybrid architecture in [1], while data logging module is developed for accurate measurements in ticks ( 1 sec. $=10^{7}$ ticks). The active hybrid architecture is selected to implement the model in-the-loop simulation, not only to avoid migration of Simulink UAV mathematical model to other environments, but also to avoid the use of auto-code generation to simplify the development, and to minimize the effort and cost.

\section{Simulation Results}

This section demonstrates the simulation results to

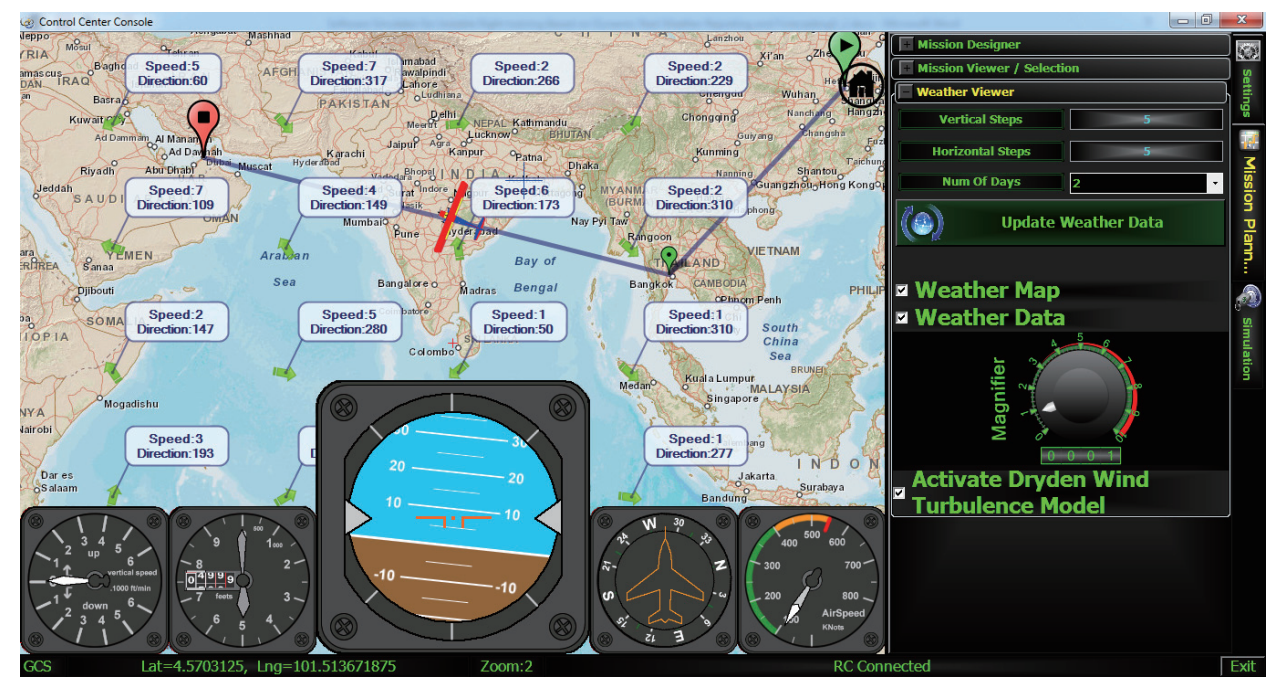

Fig. 6. Proposed Virtual Environment GUI Snapshot 
evaluate different characteristics for the proposed virtual environment, ranging from visual and vestibular capabilities to different software processing delays.

\subsection{Graphical User Interface Evaluation (Visual Ca- pabilities)}

As shown in Fig. 6, the proposed virtual environment has an innovative GUI that can simultaneously display multiple static layers (map, trajectory, waypoints...etc.) and dynamic ones (UAV symbol, gauges... etc.). The demonstrated mission starts at China, passes over Thailand, and its final destination is Dubai. The trainee loads the trajectory and requests to display weather forecast data for next two advanced days. The trainee issues guidance commands to overcome the wind turbulence along the whole trajectory. At speed 87 Knots, the real weather injected parameters fluctuate the attitude gauges $5000 \mathrm{ft}$ and let the trainee realize same feeling of UAV cockpit. It is worthily noted that the proposed virtual environment has some advanced features to enhance the simulation process such as: (1) magnifying the real weather data with any custom factor for more advanced training difficulties. (2) Presenting advanced gauges such as vertical speed, altimeter, attitude indicator, heading, and air speed. (3) Providing the recording and playback facilities. These features increase the functional fidelity [32] of the proposed platform.

Furthermore, Fig. 7 (a) demonstrates the capability of developed weather interface modules which can retrieve

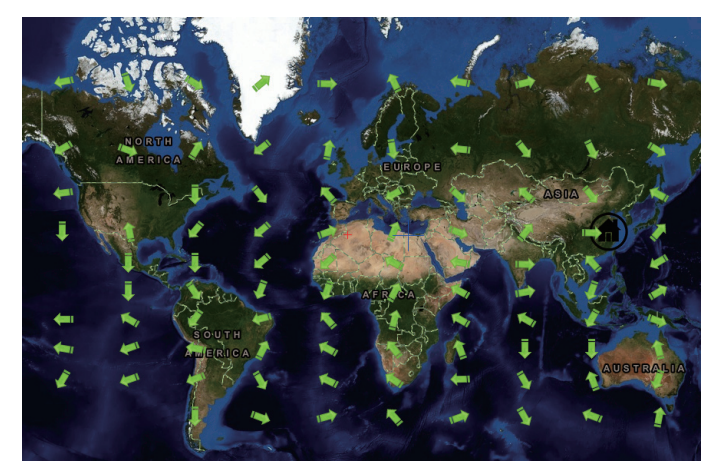

(a) the required weather data for any global coordinate, while, in Fig. 7 (b), a generated color map is illustrated to represent different longitudinal and latitudinal zones of Fig. 7 (a). The vertical color bar represents the wind speed in $\mathrm{m} / \mathrm{s}$. The color zones map could be exploited to support the off-line manual trajectory planning and automatic safe path generation. On the other hand, blue color from any source coordinate to another destination coordinate, this represents a trajectory with minimum wind turbulence (safe trajectory). Finally, it is clear that the weather integration with the proposed virtual environment plays an active role during both planning and operational phases.

\subsection{MARD Dynamics Evaluation (Vestibular Capa- bilities)}

Toward achieving behavioral results about the performance of the simulated MARD, many simulated missions have been executed.

Fig. 8 presents different time intervals of a sample mission to illustrate different MARD responses. As shown in Fig. 8 (a), a change of commanded pan angle ( $\psi$ ) from 0.2 to 0.733 (rad.) is compensated by the simulated MARD after 1.14 seconds with a delay equal to 0.11 second. In Fig. 8 (b), as an arbitrary start, the simulated MARD starts with tilt angle initial condition equal to zero (which is considered as a latency error), to provide an initial error to be compensated. The commanded $\Theta$ error has been

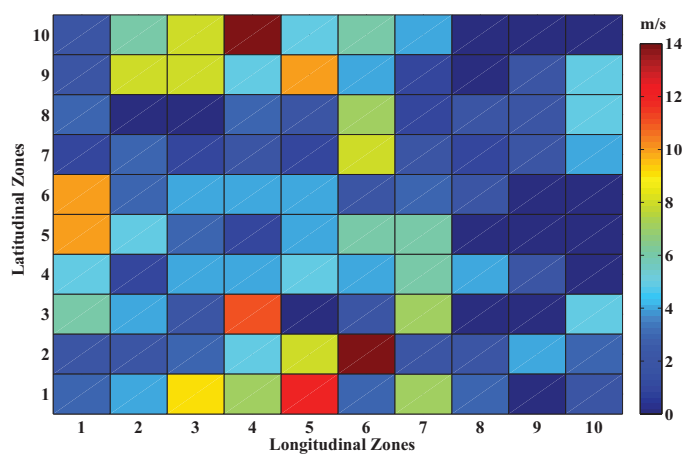

(b)

Fig. 7. The Automated Weather Interface Module. (a) Global Weather Forecast. (b) Generated Weather Color Map

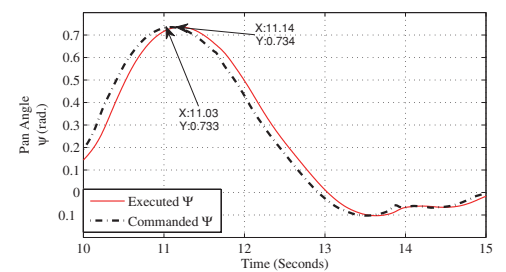

(a)

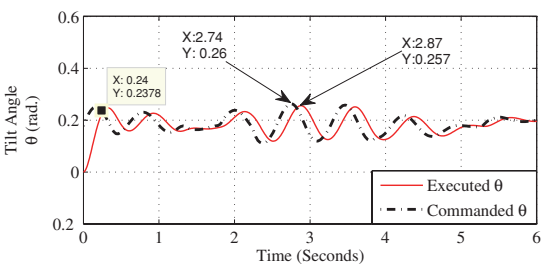

(b)

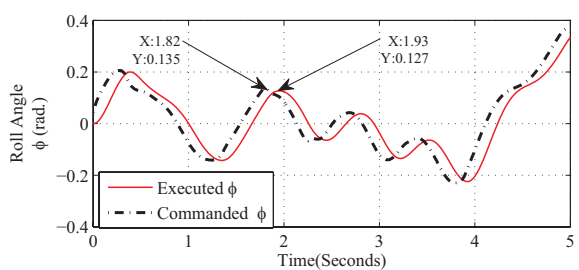

(c)

Fig. 8. MARD Responses for Different Commanded Angles 
compensated after 0.24 seconds only with an overshoot pitch less than 0.0052 rad. $\left(0.3^{\circ}\right)$. In Fig. 8 (c), the average MARD response delay is 0.11 seconds. Moreover, using the correlation between the commanded and executed angles, the average delay is calculated for different MARD axes. The average response delays for pan, tilt, and roll axis are $130 \mathrm{~ms}, 110 \mathrm{~ms}$, and $120 \mathrm{~ms}$, respectively. As a result, the total average response delay for the whole simulated MARD is the maximum of all axes average delay which is $130 \mathrm{~ms}$.

\subsection{GSM Communication Delay}

Communication delay is considered one of the most challenges for the proposed virtual environment. Thus, using short circuit between point 4 and 6 in Fig. 2 (loopback), the data logging module is adapted to compare the transmitted and received data and to measure the round trip delay. Table 1 lists a data logging sample for the loopback data transfer, which shows that the average uplink/downlink latency over 10000 samples (each sample is 29 bytes) is in order of $286 \mathrm{~ms}$, which is considered acceptable such as delays in [18].

\subsection{Different Software Modules Processing Delay}

As the proposed virtual environment relies deeply on software, thus, it is important to measure the processing delay of different data manipulation software modules. The developed data logging module is not only used for recording the exchanged data, but also for measuring the processing delay of different software module. The most critical processing delays are the interface modules which is responsible of the RC transmitter, GSM interface

Table 1. GSM Communication Delay Measurements (each sample = 29 bytes).

\begin{tabular}{lll}
\hline $\begin{array}{l}\text { Transmit } \\
\text { Time } \\
\text { (Ticks) }\end{array}$ & $\begin{array}{l}\text { Receive } \\
\text { Time } \\
\text { (Ticks) }\end{array}$ & $\begin{array}{l}\text { Round } \\
\text { Trip } \\
\text { Delay } \\
\text { (Ticks) }\end{array}$ \\
\hline 635551560641752484 & 635551560647604261 & 5851777 \\
635551560647634952 & 635551560653605129 & 5970177 \\
635551560653640420 & 635551560659494641 & 5854221 \\
635551560659532802 & 635551560665385290 & 5852488 \\
635551560665416008 & 635551560670875085 & 5459077 \\
635551560670913652 & 635551560676948354 & 6034702 \\
635551560676984607 & 635551560682878280 & 5893673 \\
635551560682916203 & 635551560688272173 & 5355970 \\
635551560688310562 & 635551560693719847 & 5409285 \\
635551560693754578 & 635551560699582413 & 5827835 \\
635551560699615279 & 635551560705263850 & 5648571 \\
635551560705294142 & 635551560710838137 & 5543995 \\
635551560641752484 & 635551560647604261 & 5851777 \\
635551560647634952 & 635551560653605129 & 5970177 \\
635551560659532802 & 635551560665385290 & 5852488 \\
\hline
\end{tabular}

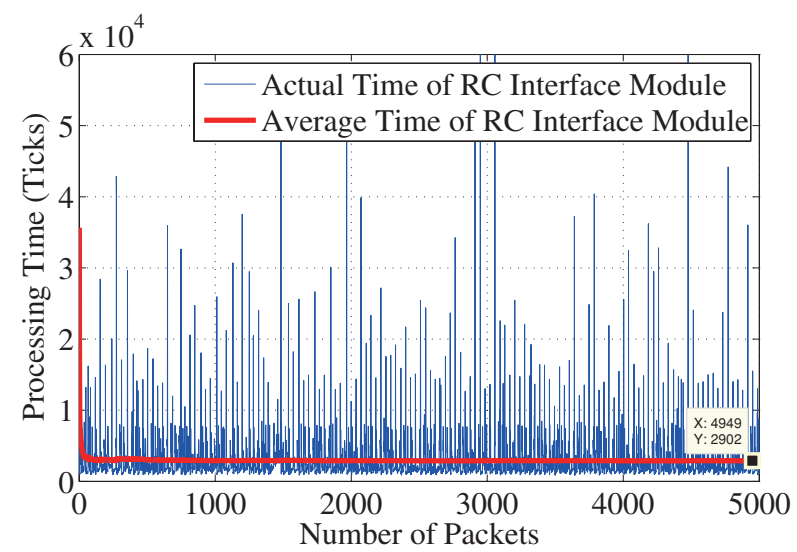

Fig. 9. Software Processing Delay of RC Interface Module (using AMD FX-8350 Black Edition 8-Core 4.0GHz)

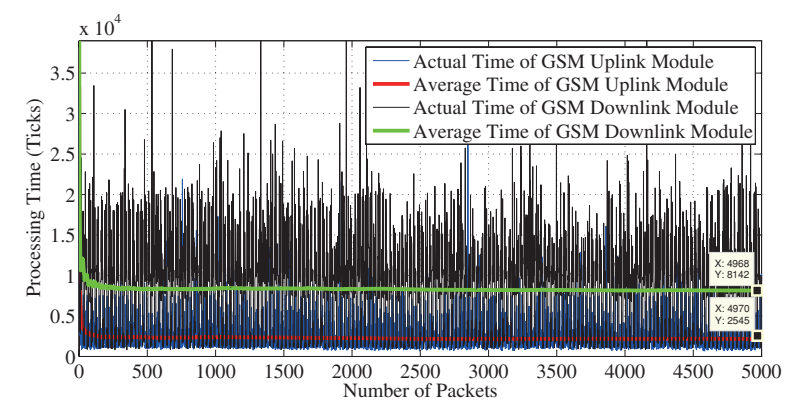

Fig. 10. Software Processing Delay of GSM Data Manipulation Module

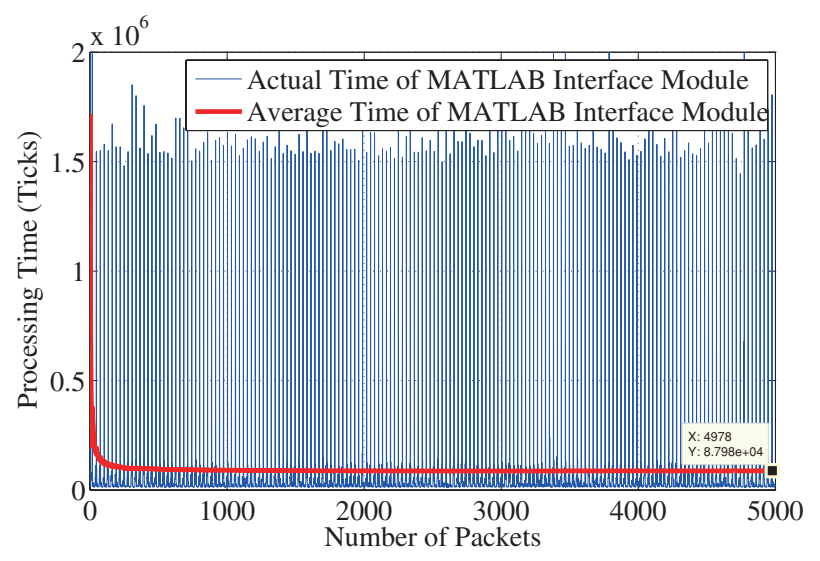

Fig. 11. Software Processing Delay of MATLAB Interface Module

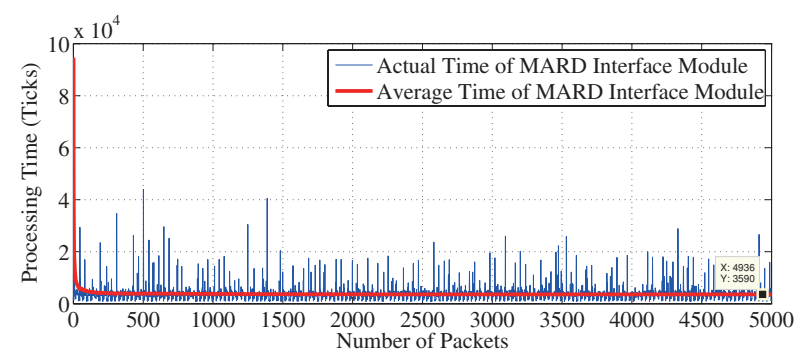

Fig. 12. Software Processing Delay of MARD Interface Module 
module, MATLAB interface module and MARD interface module. These modules are responsible of capturing the data, parsing and extracting the required parameters, and finally, submitting the data to the demanded destination. As shown in Fig. 9, the average processing delay to manipulate the RC transmitter data is 2902 ticks $(0.29 \mathrm{~ms})$. On the other hand, processing delay of GSM software interface module is divided into uplink delay (for sending UAV commands) and downlink delay (for receiving UAV flight parameters).

As shown in Fig. 10, the average processing delay of GSM uplink and downlink are 2545 ticks $(0.255 \mathrm{~ms})$, and 8142 ticks (0.815ms), respectively. Fig. 11. Demonstrates the MATLAB interface module, which represents the processing delay of MATLAB engine. From this figure, wind turbulence and aircraft mathematical model consumes 87980 ticks (8.8ms). Finally, Fig. 12 shows that MARD interface module consumes $0.35 \mathrm{~ms}$. These measurements utilize the workstation configuration in [1]. This processing delay analysis proves that the designed software modules are working efficiently and their time delay is negligible with respect to GSM propagation delay.

\section{Qualitative Aspects of the Proposed Vir- tual Environment Vs. Other Systems}

This section demonstrates some qualitative aspects, which are introduced to address the proposed virtual environment features against other systems. As the proposed virtual environment utilizes a real GSM channel, a comparison is conducted with UAV teleoperation workstations. From Table 2 , it can be noticed that the proposed virtual environment utilizes a fullscale aircraft model, utilizes a real weather

Table 2. Qualitative Aspects of the Proposed Virtual Environment and Other Systems

\begin{tabular}{|c|c|c|}
\hline Criteria & $\begin{array}{l}\text { Proposed Virtual } \\
\text { Environment }\end{array}$ & $\begin{array}{c}\text { Teleoperation } \\
\text { Systems in }[16,17]\end{array}$ \\
\hline Utilized PCs & $2 \mathrm{PC}$ & $\begin{array}{c}1 \text { PC plus } \\
1 \text { Embedded PC }\end{array}$ \\
\hline Aircraft Model & DHC-2 "Beaver" & quadcopter \\
\hline $\begin{array}{l}\text { Training Not Limited to } \\
\text { RC Range }\end{array}$ & Available & Not available \\
\hline $\begin{array}{c}\text { Synthetic Real Weather } \\
\text { Data Layer }\end{array}$ & Available & Not available \\
\hline Wind turbulence effect & $\begin{array}{l}\text { Available } \\
\text { MATLAB: }\end{array}$ & Not available \\
\hline $\begin{array}{l}\text { Simulation platform to } \\
\text { motion platform delay }\end{array}$ & $\begin{array}{c}8.7 \mathrm{~ms} \\
\text { GSM : } 286 \mathrm{~ms} \\
\text { Other Software: } \\
1.8 \mathrm{~ms}\end{array}$ & $300 \mathrm{~ms}$ \\
\hline $\begin{array}{c}\text { Individual axes max } \\
\text { delay }\end{array}$ & $130 \mathrm{~ms}$ & $148 \mathrm{~ms}$ \\
\hline Average Latency (Delay) & $426.5 \mathrm{~ms}$ & $448 \mathrm{~ms}$ \\
\hline Communication & $\begin{array}{l}\text { NLoS GSM } \\
\text { Channels }\end{array}$ & $\operatorname{LoS}$ Wireless LAN \\
\hline
\end{tabular}

data to support the teleoperator decisions, its operational range is not limited to the RC transmitter range, and finally, its total delay is similar to other teleoperation systems with same motion cues. From this comparison, it is clear that the proposed platform achieves a very long range relative to other systems which utilizes LoS communication links. Moreover, the proposed platform can be upgraded to a functional teleoperation workstation with minor modifications.

\section{Conclusion}

This paper proposed a new UAV virtual environment to enhance the pilot expertise and to advance the situational awareness during real time operation of a UAV that is beyond LoS. The proposed platform considers real GSM communication channels, realistic wind turbulence parameters based-on real weather data, and a simulated model for an existing UAV and MARD for better evaluation. The results show that the proposed virtual environment provides the required motion cues to teleoperator via GSM network to control a UAV beyond LoS with latency less than $430 \mathrm{~ms}$. These results are considered acceptable relative to other teleoperation systems, which uses LoS communications. These encouraging results provide a good motivation towards upgrading the proposed virtual environment to be a functional long range teleoperation GCS with visual and vestibular feedbacks, which will extend the range of medium altitude UAVs.

\section{Acknowledgement}

The authors would like to acknowledge the anonymous reviewers for their insightful comments and suggestions that leads to significant enhancement in the paper quality. In addition, the authors would like to thank the Chinese government "National 863 fund" (ref. 2014AA7010051) for their great support.

\section{References}

[1] A. AbdElHamid, and P. Zong, "A Novel Software Simulator Model Based on Active Hybrid Architecture", International Journal of Aerospace Engineering, Vol. 2015, 2015, pp. 19. DOI:10.1155/2015/107301

[2] R. Rodrigues, R. C. B. Sampaio, A. P. Aguiar, and M. Becker, "FVMS Software-in-the-Loop Flight Simulation Experiments: Guidance, Navigation and Control", Proceeding 
of 2014 Joint Conference on Robotics: SBR-LARS Robotics Symposium and Robocontrol (SBR LARS Robocontrol), San Carlos, Sao Paulo, 2014, pp. 223-228.

[3] S. Zheng, Q. Huang, J. Jin, and J. Han, "Flight Simulator Architecture Development and Implementation", Proceeding of 2009. ICMTMA '09. International Conference on Measuring Technology and Mechatronics Automation, Zhangjiajie, Hunan, 2009, pp. 230-233.

[4] P. Kemao, M. C. Ben, and L. Kai Yew, "Autonomous Flight Control Design for a Small-scale Unmanned Helicopter", in AIAA Guidance, Navigation, and Control Conference, American Institute of Aeronautics and Astronautics, 2011.

[5] S. Tang, X. Lu, and Z. Zheng, "Platform and State Estimation Design of a Small-Scale UAV Helicopter System", International Journal of Aerospace Engineering, Vol. 2013, 2013, pp. 13. DOI:10.1155/2013/524856

[6] Miaobo Dong, Ben M. Cheni, Guowei Cai, and K. Peng, "Development of a Real-time Onboard and Ground Station Software System for a UAV helicopter", Journal of Aerospace Computing, Information, and Communication, Vol. 4, No. 8, 2007, pp. 933-955.

[7] Y. Fan, Z. Zhang, M. Trinkle, A. D. Dimitrovski, J. B. Song, and H. Li, "A Cross-Layer Defense Mechanism Against GPS Spoofing Attacks on PMUs in Smart Grids", IEEE Transactions on Smart Grid, Vol. PP, No. 99, 2014, pp. 1-10. DOI:10.1109/tsg.2014.2346088

[8] G. Shuping, Z. Zhenghao, M. Trinkle, A. D. Dimitrovski, and L. Husheng, "GPS spoofing based time stamp attack on real time wide area monitoring in smart grid", Proceeding of 2012 IEEE Third International Conference on Smart Grid Communications (SmartGridComm), Tainan, Taiwan, 2012, pp. 300-305.

[9] L. Khoa Duy, N. Hung Duc, D. Ranthumugala, and A. Forrest, "Haptic driving system for surge motion control of underwater remotely operated vehicles", Proceeding of the 6th International Conference on Modelling, Identification \& Control (ICMIC), 2014, Melbourne, Australia, pp. 207-212.

[10] F. Ryden, A. Stewart, and H. J. Chizeck, "Advanced telerobotic underwater manipulation using virtual fixtures and haptic rendering", Proceeding ofOceans - San Diego, 2013, pp. 1-8.

[11] A. M. Brandt, and M. B. Colton, "Haptic collision avoidance for a remotely operated quadrotor UAV in indoor environments", Proceeding of IEEE International Conference on Systems Man and Cybernetics (SMC), Istanbul, Turkey, 2010, pp. 2724-2731.

[12] L. Dongjun, A. Franchi, P. R. Giordano, S. Hyoung Il, and H. H. Bulthoff, "Haptic teleoperation of multiple unmanned aerial vehicles over the internet", Proceeding of 2011 IEEE International Conference on Robotics and Automation (ICRA), Shanghai, China, 2011, pp. 1341-1347.
[13] T. M. Lam, M. Mulder, and M. M. van Paassen, "Collision avoidance in UAV tele-operation with time delay", Proceeding of 2007 IEEE International Conference on Systems, Man and Cybernetics, Montreal, QC, 2007, pp. 997-1002.

[14] J. Borenstein, and Y. Koren, "Real-time obstacle avoidance for fast mobile robots in cluttered environments", Proceeding of 1990 IEEE International Conference on Robotics and Automation, Cincinnati, Ohio, 1990, pp. 572-577.

[15] L. Sangyoon, G. Sukhatme, K. Jounghyun, and P. Chan-Mo, "Haptic control of a mobile robot: a user study", Proceeding of 2002. IEEE/RSJ International Conference on Intelligent Robots and Systems, EPFL Lausanne, Switzerland, 2002, pp. 2867-2874.

[16] T. Harald, N. Hans-Günther, B. Karl, B. John, K. Michael, and B. Heinrich, "MPI Motion Simulator: Development and Analysis of a Novel Motion Simulator", in AIAA Modeling and Simulation Technologies Conference and Exhibit, American Institute of Aeronautics and Astronautics, 2007.

[17] J. Hing, and P. Oh, "Development of an Unmanned Aerial Vehicle Piloting System with Integrated Motion Cueing for Training and Pilot Evaluation", in Unmanned Aircraft Systems, Springer Netherlands, 2009, pp. 3-19.

[18] P. R. Giordano, H. Deusch, J. Lächele, and H. H. Bülthoff, "Visual-vestibular feedback for enhanced situational awareness in teleoperation of UAVs", Proceeding of AHS 66th Annual Forum and Technology Display, South Carolina, 2010.

[19] J. T. Hing and P. Y. Oh, "Mixed reality for unmanned aerial vehicle operations in near Earth environments", Proceeding of 2010 IEEE/RSJ International Conference on Intelligent Robots and Systems (IROS), Taipei, Taiwan, 2010, pp. 2537-2538.

[20] J. M. Teixeira, R. Ferreira, M. Santos, and V. Teichrieb, "Teleoperation Using Google Glass and AR, Drone for Structural Inspection", Proceeding of 2014 XVI Symposium on Virtual and Augmented Reality (SVR), Salvador, Brazil, 2014 pp. 28-36.

[21] Frederick R Carrick, Guido Pagnacco, Elena Oggero, Stephanie Sullivan, Derek Barton, Susan Esposito, Gerry Leisman, and R. Melillo, "The Effects of Whole Body Rotations in the Pitch and Yaw Planes on Postural Stability", Functional Neurology, Rehabilitation, and Ergonomics, Vol. 1, No. 2, 2011, pp. 167-180.

[22] S. Ronald, F. Alia, K. John, and W. Christopher, "A Pilot Spatial Orientation Aiding System", in AIAA 5th ATIO and16th Lighter-Than-Air Sys Tech. and Balloon Systems Conferences, American Institute of Aeronautics and Astronautics, 2005.

[23] J. T. Hing, and P. Y. Oh, "A motion platform integrated uav pilot training and evaluation system for future civilian applications", Proceeding of ASME 2008 Dynamic Systems 
and Control Conference, Ann Arbor, Michigan, USA, 2008, pp. 447-454.

[24] M. Rauw, FDC 1.2 - A Simulink Toolbox for Flight Dynamics and Control Analysis, 2nd edition, 2001.

[25] L. Manmaker, MATLAB External Interfaces, The MathWorks, 2013.

[26] The MathWorks, Inc., Application Program Interface Guide, Version 5, The MathWorks, Inc., 1998,

[27] E. F. Hogge, B-737 linear autoland simulink model, NASA, Langley Research Center, Hampton, Virginia, USA, Tech. Rep. CR-2004-213021, 2004.

[28] S. Fortunati, A. Farina, F. Gini, A. Graziano, M. S. Greco, and S. Giompapa, "Impact of flight disturbances on airborne radar tracking", IEEE Transactions on Aerospace and Electronic Systems, Vol. 48, No. 3, 2012, pp. 2698-2710.

[29] D. Moorhouse, and R. Woodcock, US Military Specification MIL-F-8785C, Technical report, US Department of Defense, 1980.

[30] The MathWorks, Inc., Dryden Wind Turbulence Model, MATLAB Reference Pages, 2010,

[31] F. Hargrave, Appendixes A:AT Fax/Modem Commands, Wiley-IEEE Press, 2001.

[32] J. Craighead, R. Murphy, J. Burke, and B. Goldiez, "A Survey of Commercial \& Open Source Unmanned Vehicle Simulators", Proceeding of IEEE International Conference on Robotics and Automation, Roma, Italy, 2007, pp. 852-857. 